\title{
Comparison of paper-based nucleic acid extraction materials for point-of-care testing applications
}

\author{
Ruihua Tang $\cdot$ Min Li $\cdot$ Xueyan Yan $\cdot$ Mingyue Xie $\cdot$ Li Na Liu $\cdot$ \\ Zedong Li $\cdot$ Feng $\mathrm{Xu}$
}

Received: 4 October 2021 / Accepted: 18 January 2022 / Published online: 29 January 2022

(C) The Author(s), under exclusive licence to Springer Nature B.V. 2022

\begin{abstract}
Cheap, rapid, simple and equipment-free nucleic acid extraction (NAE) is highly preferred for implementing nucleic acid detection at point-ofcare (POC). Paper-based NAE materials have been extensively utilized due to their low cost, abundance, portability, biocompatibility and ease of chemical modification. However, it is challenging for users to choose the proper one from existing paper-based NAE materials for specific POC applications, which is determined by their physical and chemical properties. Additionally, building the relationship between the physical and chemical properties and the NAE efficiency of paper-based materials is instructive for development of new paper-based NAE materials. In
\end{abstract}

Supplementary Information The online version contains supplementary material available at https://doi.org/10.1007/ s10570-022-04444-6.

R. Tang $\cdot$ Z. Li $(\bowtie) \cdot$ F. Xu $(\bowtie)$

The Key Laboratory of Biomedical Information

Engineering of Ministry of Education, School of Life

Science and Technology, Xi' an Jiaotong University,

Xi'an 710049, People's Republic of China

e-mail: zedong@mail.xjtu.edu.cn

F. Xu

e-mail: fengxu@mail.xjtu.edu.cn

R. Tang $\cdot$ M. Li $\cdot$ X. Yan $\cdot$ M. Xie

College of Bioresources Chemical and Materials

Engineering, Shaanxi University of Science \&

Technology, Xi' an 710021, People's Republic of China this study, we first systematically compared the physical and chemical properties of six widely used paper-based NAE materials (namely Whatman filter paper \#1, FTA card, FTA elute card, Fusion 5, silica membrane and polyethersulfone (PES) membrane), and then evaluated their NAE efficiency. The obtained results indicated that pore uniformity, wet strength, porosity and functional groups are key parameters to affect the efficiency of NAE. The NAE performance of FTA card is the best with high concentration and purity. Finally, we envision that more cost-effective paper-based NAE materials will be developed for POCT application in the future. 


\section{Graphic abstract}

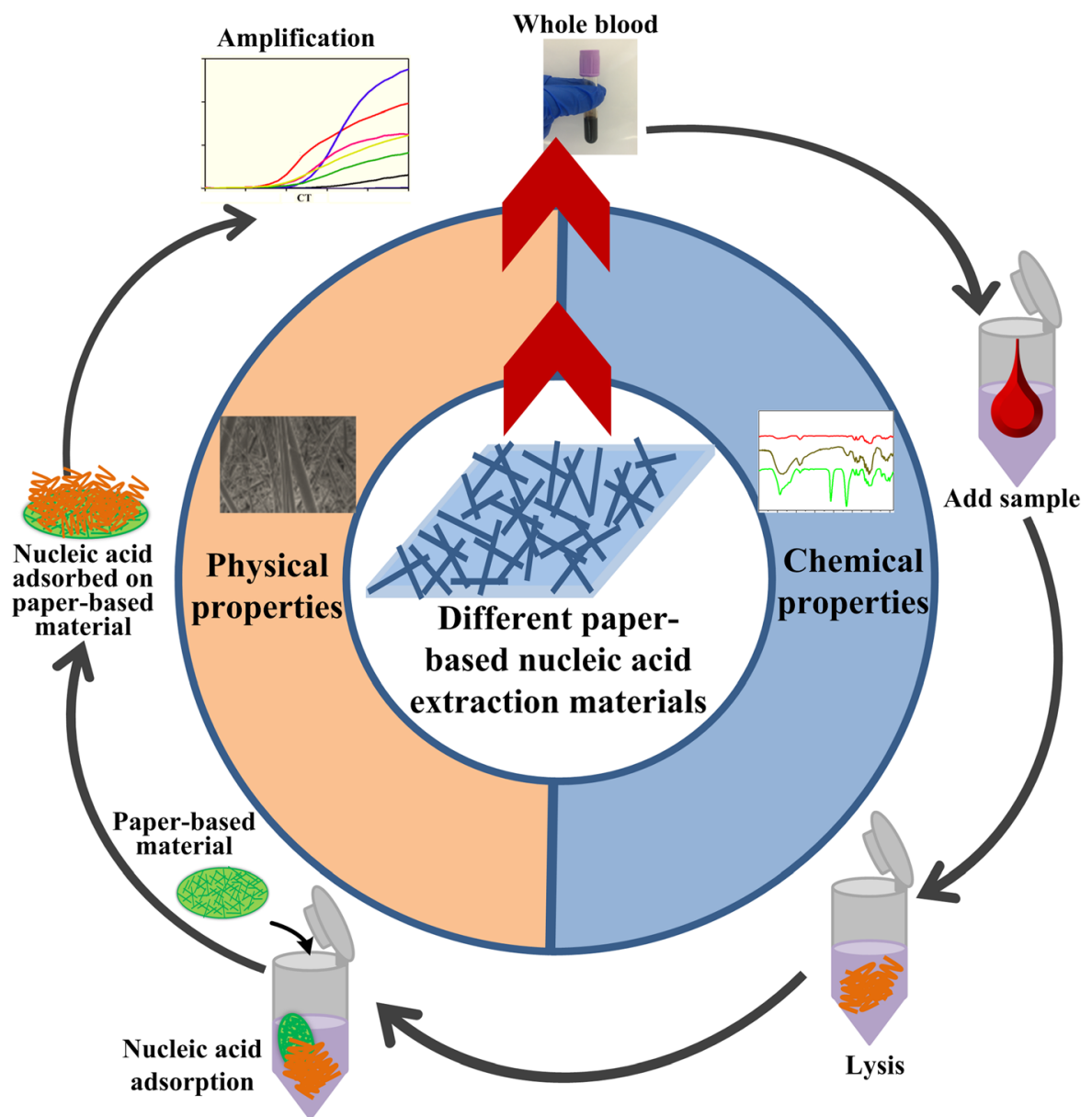

The performance of nucleic acid extraction

Keywords Cotton fiber - Glass fiber - PES membrane $\cdot$ Nucleic acid extraction $\cdot$ Paper-based material

\section{Introduction}

Nucleic acid detection at point-of-care (POC) as a highly specific and sensitive diagnostic technology has been widely used in medical field, such as infectious diseases screening (Li et al. 2021c), cancer genetics diagnosis (Yang et al. 2021), host-pathogen interaction (Mika-Gospodorz et al. 2020), bacterial and virus testing (Yi et al. 2021). Especially, since the outbreak of COVID-19 pandemic at 2019, this technology has been chosen as the standard diagnostic tool to screen and control the infectious condition of COVID-19 in the world (Xun et al. 2021). Nucleic acid extraction (NAE) is the primary step for nucleic acid detection, which determines the success in downstream amplification and signal analysis (Li et al. 2021a). Traditional NAE methods, such as phenol extraction method (Emaus et al. 2020), spin-column method (Li et al. 2021b) and magnetic bead method (Chen et al. 2020), are often associated with the issues of toxic reagents, bulky equipment, skilled operator, complex and time-consuming ( $40 \mathrm{~min}$ to $1 \mathrm{~h}$ ) process. Thus, it is necessary to develop a cheap, rapid, simple and 
equipment-free NAE technology for carrying out nucleic acid detection at point-of-care.

Paper, as a low-cost, abundant, portable, biocompatible and easy-to-modify material, has been widely used for fabricating paper-based NAE device (Batule et al. 2020; Mason and Botella 2020), which exhibits satisfactory performance for enabling nucleic acid detection at POC due to ease of use and cold chain-free transfer and storage. Common paper-based NAE devices are mainly based on various types of paperbased materials through nucleic acid specific adsorption and desorption, including Whatman filter paper \#1 (Ghosh and Hazarika 2018; Hashimoto et al. 2019; Zou et al. 2017), FTA card (Choi et al. 2016), FTA elute card (Hashimoto et al. 2019), Fusion 5 (Jangam et al. 2009), silica membrane (Tang et al. 2019) and polyethersulfone (PES) membrane (Mandal et al. 2018; Rodriguez et al. 2015). For instance, Fusion 5-based automated DNA extraction device can extract DNA from whole blood within 8 min (Gan et al. 2014). FTA card can extract DNA from whole blood, drinking water, milk, spinach (Choi et al. 2016) and swab within 40 min (Chauhan et al. 2021). FTA elute card can extract DNA from dried blood spots (Hashimoto et al. 2019). Silica membrane integrated into paper-based device can extract DNA from foodborne pathogens within 12 min (Wang et al. 2020). The main difference in their discriminative applications is attributed to different paper-based materials with various physical properties (e.g., porosity and mechanical strength) and chemical properties (e.g., surface element and functional group) (Table 1), which are closely related to their performances. For instance, the special dielectric, piezoelectric high aspect ratio, good wettability and thermal stability enable cellulose-based material to possess flexibility and conductivity (Zhao et al. 2020). Thus, studying their physical and chemical properties is of great significance for users to choose the proper one for specific application. Besides, building the relationship between the physical and chemical properties and the NAE efficiency of these paper-based materials is instructive for development of new paperbased NAE technologies.

In this study, we compared the performance of six common paper-based NAE materials, including physical properties, chemical properties and NAE efficiencies. Firstly, we chose six types of existing commercial paper-based NAE materials (i.e., Whatman filter paper \#1, FTA card, FTA elute card, Fusion 5, PES membrane, silica membrane). Next, their physical properties (i.e., the type and quality of fiber, morphology, mechanical strength and hydrophilicity) and chemical properties (i.e., surface element, functional group and crystal structure) were investigated. Finally, whole blood as a model sample was used to evaluate NAE efficiency of these materials.

\section{Materials and experiments}

\section{Materials}

Whatman filter paper \#1 (diameter of $125 \mathrm{~mm}$ ), FTA card, FTA elute card, Fusion 5 and FTA purification reagent were purchased from GE Healthcare Life Science (USA). Express ${ }^{\circledR}$ Plus PES membrane was

Table 1 Inclusion and exclusion criteria of paper-based nucleic acid extraction material applied for point-of-care testing in existing literature

\begin{tabular}{ll}
\hline Inclusion criteria & Exclusion criteria \\
\hline $\begin{array}{l}\text { Know physical property (e.g., pore size, porosity, thickness, mechanical } \\
\text { strength.) }\end{array}$ & $\begin{array}{c}\text { Few studies know pore size and thickness, most studies } \\
\text { not clear } \\
\text { unclear }\end{array}$ \\
Know chemical property (e.g., functional group, surface element.) & Few type is cheap (e.g., glass fiber-based material) \\
Low cost & $\begin{array}{l}\text { It still needs skilled workers } \\
\text { Simplified operation }\end{array}$ \\
$\begin{array}{l}\text { Portability } \\
\text { High efficiency } \\
\text { Integrated functional material including sample lysis, nucleic acid } \\
\text { adsorption and non-specific substance washing }\end{array}$ & $\begin{array}{l}\text { Different materials have different efficiencies } \\
\text { Existing studies mainly focus on developing various } \\
\text { paper-based nucleic acid device }\end{array}$ \\
\hline
\end{tabular}


obtained from Merck Millipore Ltd. (USA). Silica membrane was obtained from Laifeng Biotech Co., Ltd. (Hangzhou, China). QIAamp® DNA Micro kit was purchased from Qiagen (German). Guanidinium thiocyanate (GuSCN), Tween-20, Triton X-100 and isopropanol were purchased from Amersco (USA). Proteinase K was bought from Tiangen Biotechnology Co., Ltd. (Beijing, China). Ethylene diamine tetraacetic acid (EDTA), sodium dodecyl sulfate (SDS), tris-hydroxymethyl aminomethane (Tris), sodium hydroxide and zinc chlorides were purchased from Damao Chemical Reagent Co., Ltd. (Tianjin, China). Iodine granules were bought from Kemi Chemical Reagent Co., Ltd. (Tianjin, China). Guanidine hydrochloride was obtained from Shanghai Aladdin Biotech Co., Ltd (China). Ethanol was purchased from Sinopharm Chemical Reagent Co., Ltd. (China). TE buffer solution was purchased from Generay Biotech Co., Ltd. (Shanghai, China). Nucleic acid primers were obtained from Sangon Biotech Co., Ltd. (Shanghai, China). TB Green ${ }^{\circledR}$ Premix Ex Taq $^{\mathrm{TM}}$ was purchased from Baori Biotechnology Co., Ltd. (Beijing, China). All reagents were analytical purity. Whole blood sample was from the First Affiliated Hospital of Xi'an Jiaotong University. Informed consent for research use of whole blood was sought and obtained from the participant.

Characterization of paper-based nucleic acid extraction materials

\section{Fiber type}

Fiber types of six paper-based NAE materials were observed using high-power optical microscopy. The preparation process of sample is as follows: paperbased material was soaked and dispersed in water; Next, the dispersed fibers were placed on a piece of glass slide and dyed with Iodine-zinc chlorides reagent (Herzberg reagent); Finally, the fiber type was determined by optical microscopy based on color of fiber. The average width and branching rate of fiber (\%) were measured by the fiber quality analyzer (Techpap, Morfi Compact, France).

\section{Physical properties}

The thickness of paper-based materials was measured by the thickness gauge (313-a, High Speed Rail
Technology Co., Ltd., China). The quantity and tightness of paper-based materials were calculated by Eqs. (1) and (2), respectively. The morphology, including front section, back section and cross section, was investigated by scanning electron microscopy (SEM) (HITACHI Co. Ltd., Japan). The dry tensile strength and wet strength of paper-based materials were analyzed by servo material multi-function tester (AI-7000-NGD, Goodtechwill, China). The shape changes of paper-based materials before and after immersion in water was investigated by smart phone (iPhone 6S). The hydrophilicity and porosity of paperbased materials were evaluated by video-based contact angle device (OCR20, Germany) and mercury porosimeter (Micromeritics, Auto Pore IV 9500, USA), respectively.

Paper quantity $=\frac{\text { Paper quality }}{\text { Paper area }}$

Tightness $=\frac{\text { Paper quantity }}{\text { Paper thickness }}$

\section{Chemical properties}

The surface elements of paper-based materials were analyzed by energy-dispersive x-ray spectroscopy (EDS) (SU8100, HITACHI Co. Ltd., Japan). The surface functional groups of paper-based materials were analyzed by Fourier transforms infrared spectroscopy (FTIR) (Vertex 70, Netzsch, Germany) and $\mathrm{X}$-ray photoelectron spectroscopy (XPS) (Thermo Scientific K-Alpha+, ThermoFisher, USA). The crystal structure of paper-based materials was analyzed by X-ray diffractometer (XRD) (Bruker, D8 Advance, Germany).

Preparation of paper-based materials for nucleic acid extraction

Six paper-based NAE materials (Whatman filter paper \#1, FTA card, FTA elute card, Fusion 5, silica membrane and PES membrane) were firstly cut into disk shape with a diameter of $4.5 \mathrm{~mm}$ through punching.

The Whatman filter paper \#1 was prepared according to the previous study (Zou et al. 2017) with slight modification. $30 \mu \mathrm{L}$ of whole blood, $3 \mu \mathrm{L}$ of proteinase $\mathrm{K}$ and $120 \mu \mathrm{L}$ of extraction buffer ( $800 \mathrm{mM}$ guanidine 
hydrochloride, $50 \mathrm{mM}$ Tris $(\mathrm{pH} 8.0), 0.5 \%$ Triton $\mathrm{X}-100,1 \%$ Tween-20) were firstly mixed in EP tube for $1 \mathrm{~min}$. The whole solution passed through the disk of Whatman filter paper \#1 and the disk was transferred into the $200 \mu \mathrm{L}$ of washing buffer (10 $\mathrm{mM}$ Tris, $\mathrm{pH}$ 8.0) for $1 \mathrm{~min}$, and then the disk was directly used for the downstream analysis.

According to the instruction, the extracted process of FTA card was as follows: $30 \mu \mathrm{L}$ of whole blood was added on the disk in the EP tube. Next, the disk was washed with $750 \mu \mathrm{L}$ of FTA purification buffer for three times. Then, it was washed with $750 \mu \mathrm{L}$ of TE buffer (10 mM Tris, $\mathrm{pH}$ 8.0) for two times. After washing, the disk was directly used for the downstream analysis.

According to the instruction, the extracted process of FTA elute card was as follows: $30 \mu \mathrm{L}$ of whole blood was added on the disk in EP tube. Then, the disk was rinsed by $750 \mu \mathrm{L}$ of $\mathrm{ddH}_{2} \mathrm{O}$ and vortexed three times for $5 \mathrm{~s}$ and centrifuged to remove water. Then, $13 \mu \mathrm{L}$ of $\mathrm{ddH}_{2} \mathrm{O}$ was added on the disk in the EP tube and it was heated at $95{ }^{\circ} \mathrm{C}$ for $30 \mathrm{~min}$. After removing the disk, it was gently tapped about 60 times. Finally, the nucleic acid elution buffer was obtained by centrifuged for $30 \mathrm{~s}$, which was used for the downstream analysis.

According to the reported literature (Tang et al. 2017) with slight modification, the extracted process on Fusion 5 and silica membrane was as follows: 30 $\mu \mathrm{L}$ of whole blood was lysed by $200 \mu \mathrm{L}$ of $5 \%$ TritonX-100 and then the whole solution flowed through the disk; Next, the disk was washed by 400 $\mu \mathrm{L}$ of $50 \mathrm{mM} \mathrm{NaOH}$; Finally, the disk was directly used for downstream analysis.

The PES membrane was prepared according to the reported literature (Mandal et al. 2018) with slight modification. $30 \mu \mathrm{L}$ of whole blood, $3 \mu \mathrm{L}$ of protein $\mathrm{K}$ and $30 \mu \mathrm{L}$ of lysis buffer ( $30 \mathrm{mM}$ Tris- $\mathrm{Cl}, \mathrm{pH} 8.0,50$ mM EDTA, 5\% Tween 20, 0.5\% Triton X-100, $1 \mathrm{M}$ GuSCN) were added in EP tube. After mixing, this tube was incubated at $56^{\circ} \mathrm{C}$ for $40 \mathrm{~min}$. Then, $63 \mu \mathrm{L}$ of $100 \%$ ethanol was added in the EP tube. After reverse mixed, this whole solution passed through the disk. Next, $150 \mu \mathrm{L}$ of washing buffer ( $2 \mathrm{M} \mathrm{GuSCN}, 60 \%$ isopropanol), $150 \mu \mathrm{L}$ of $70 \%$ frozen ethanol and 150 $\mu \mathrm{L}$ of $100 \%$ ethanol were successively used to wash the disk for two times. After drying for $5 \mathrm{~min}, 13 \mu \mathrm{L}$ of Tris-EDTA (10 mM Tris-Cl and 1 mM EDTA, pH 8.0) was added on the disk to incubate at room temperature for $2 \mathrm{~h}$. Finally, the nucleic acid elution buffer was obtained by gently tapped, which was used for downstream analysis.

For Qiagen DNA kit, the extracted process was slight modified with its instruction. $30 \mu \mathrm{L}$ of whole blood, $3 \mu \mathrm{L}$ of protein $\mathrm{K}$ and $187 \mu \mathrm{L}$ of PBS were firstly mixed in EP tube. Next, $30 \mu \mathrm{L}$ of AL buffer was added into the EP tube, and vortexed and heated in water at $56{ }^{\circ} \mathrm{C}$ for $10 \mathrm{~min} .30 \mu \mathrm{L}$ of sewage ethanol was added in the EP tube and then vortexed. Then, this mixture solution was transferred into Dneasy mini column and placed in $2 \mathrm{~mL}$ EP tube. After centrifugation for $1 \mathrm{~min}$, the waste liquid was discarded. The Dneasy mini column was transferred into a new $2 \mathrm{~mL}$ EP tube and $75 \mu \mathrm{L}$ of AW2 was added on it. After centrifugation for $3 \mathrm{~min}$, the Dneasy mini column was transferred into another new $2 \mathrm{~mL}$ EP tube, and $13 \mu \mathrm{L}$ of elution buffer was added on the membrane of nucleic acid adsorption for $1 \mathrm{~min}$ at room temperature. After centrifugation for $1 \mathrm{~min}, 13 \mu \mathrm{L}$ of DNA solution was directly utilized for downstream analysis.

Efficiency evaluation of paper-based materials for nucleic acid extraction

Real time PCR (RT-PCR) and PCR were used to verify the success of NAE and evaluate the NAE efficiency of six paper-based materials. The primers and amplification production sequences of GDPAH (human genes) were supplied in Table S1. For the standard curve, the sample was prepared by QIAamp ${ }^{\circledR}$ DNA Micro kit, and the concentration of this DNA was measured by NanoReady Micro-Volume Spectrophotometer (FC-3100, Lifereal Biotech Co., Ltd., Hangzhou, China). Then, different concentrations of samples $\left(6 \times 10^{3}, 6 \times 10^{2}, 6 \times 10^{1}, 6 \times 10^{0} \mathrm{ng} / \mathrm{mL}\right.$, negative) were prepared as standard samples. $30 \mu \mathrm{L}$ of the amplification system contains $1 \mu \mathrm{L}$ of $50 \mu \mathrm{M}$ forward primers, $1 \mu \mathrm{L}$ of $50 \mu \mathrm{M}$ reverse primers, $15 \mu \mathrm{L}$ of TB Green ${ }^{\circledR}$ Premix Ex TaqTM, $10 \mu \mathrm{L}$ DNA template solution and $3 \mu \mathrm{L}$ of $\mathrm{ddH}_{2} \mathrm{O}$. RT-PCR was evaluated by $\mathrm{CT}$ values. For six paper-based NAE materials, 30 $\mu \mathrm{L}$ of the amplification system contains $1 \mu \mathrm{L}$ of $50 \mu \mathrm{M}$ forward primers, $1 \mu \mathrm{L}$ of $50 \mu \mathrm{M}$ reverse primers, 15 $\mu \mathrm{L}$ of TB Green ${ }^{\circledR}$ Premix Ex TaqTM, DNA sample on paper disk (i.e., Whatman filter paper \#1, FTA card, Fusion 5 and silica membrane) and $13 \mu \mathrm{L}$ of $\mathrm{ddH}_{2} \mathrm{O}$; For amplification on FTA elute card, PES membrane 
and Qiagen DNA kit, $13 \mu \mathrm{L}$ of DNA solution was used.

In addition, PCR was used to verify the extracted success of six paper-based NAE materials and Qiagen DNA kit. These primers were the same with that of RT-PCR. For six paper-based NAE materials and Qiagen DNA kit, $30 \mu \mathrm{L}$ of the amplification system was as follows: $1 \mu \mathrm{L}$ of $50 \mu \mathrm{M}$ forward primers, $1 \mu \mathrm{L}$ of $50 \mu \mathrm{M}$ reverse primers, $15 \mu \mathrm{L}$ of Premix Ex TaqTM, DNA on paper disk (Whatman filter paper \#1, FTA card, Fusion 5 and Silica membrane) and $13 \mu \mathrm{L}$ of $\mathrm{ddH}_{2} \mathrm{O}$ were used; For FTA elute card, PES membrane and Qiagen DNA kit, $13 \mu \mathrm{L}$ of DNA solution was used. The amplification production was investigated by gel electrophoresis.

\section{Results and discussion}

To facilitate users to choose the proper material, in this study, we compared the performance of six paperbased materials, including Whatman filter paper \#1, FTA card, FTA elute card, Fusion 5, silica membrane and PES membrane (Fig. 1). The physical and chemical properties of these paper-based materials were firstly investigated and then their NAE performances were also observed.
Physical properties of paper-based nucleic acid extraction materials

To understand the raw materials of six paper-based NAE materials, the fiber types of these materials were firstly observed by optical microscopy after fiber dyeing (Fig. 2). The results showed that the fibers of Whatman filter paper \#1 (Fig. 2Aa), FTA card (Fig. 2Ab), FTA elute Card (Fig. 2Ac) are wine red color with no pits and miscellaneous; the average width of cotton fiber is longer than that of glass fiber (Fig. 2Ba); the branching rate of cotton fiber (\%) without modification is higher than that of cotton fiber with modification (Fig. 2Bb), which shows the obvious filament splitting (labeled with "“6( )")"). These results indicated that the raw materials of the three papers are cotton fiber. Conversely, most fibers of Fusion 5 (Fig. 2Ad) and silica membrane (Fig. 2Ae) are colorless and straight, and few fibers of the two papers are soft and curved, indicating that the raw materials of the two papers contain glass fiber (Etcheverry and Barbosa 2012) and other fiber. For PES membrane, this material is difficult to disperse in water and unable to dye. We only observed the black agglomerate from optical microscopy (Fig. 2Af), indicating that the raw material is not fiber, so it may be a polymer (Jiang et al. 2018).

To further understand the physical properties of six paper-based NAE materials, the quantity, thickness, tightness and hydrophilicity of these materials were

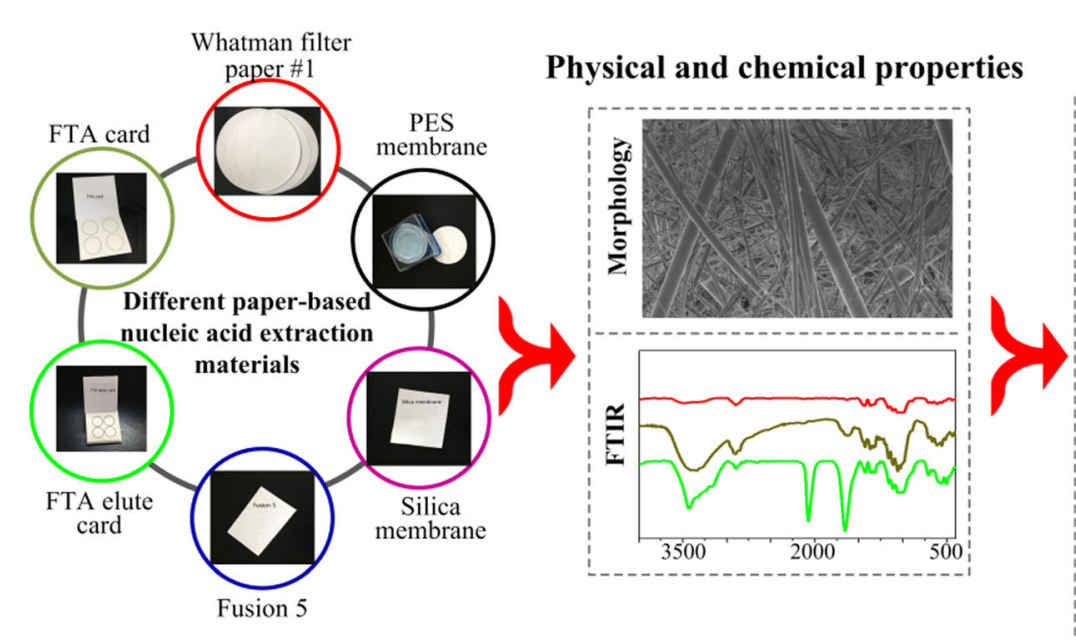

Physical and chemical properties

The performance of nucleic acid extraction

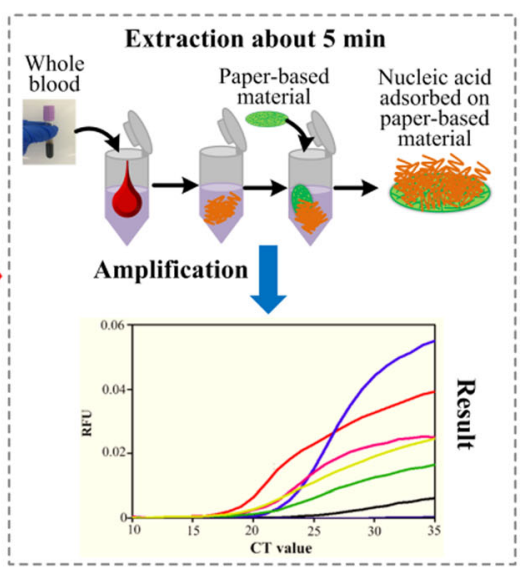

Fig. 1 Comparison of the performance of existing commercial paper-based nucleic acid extraction materials for point-of-care testing applications 

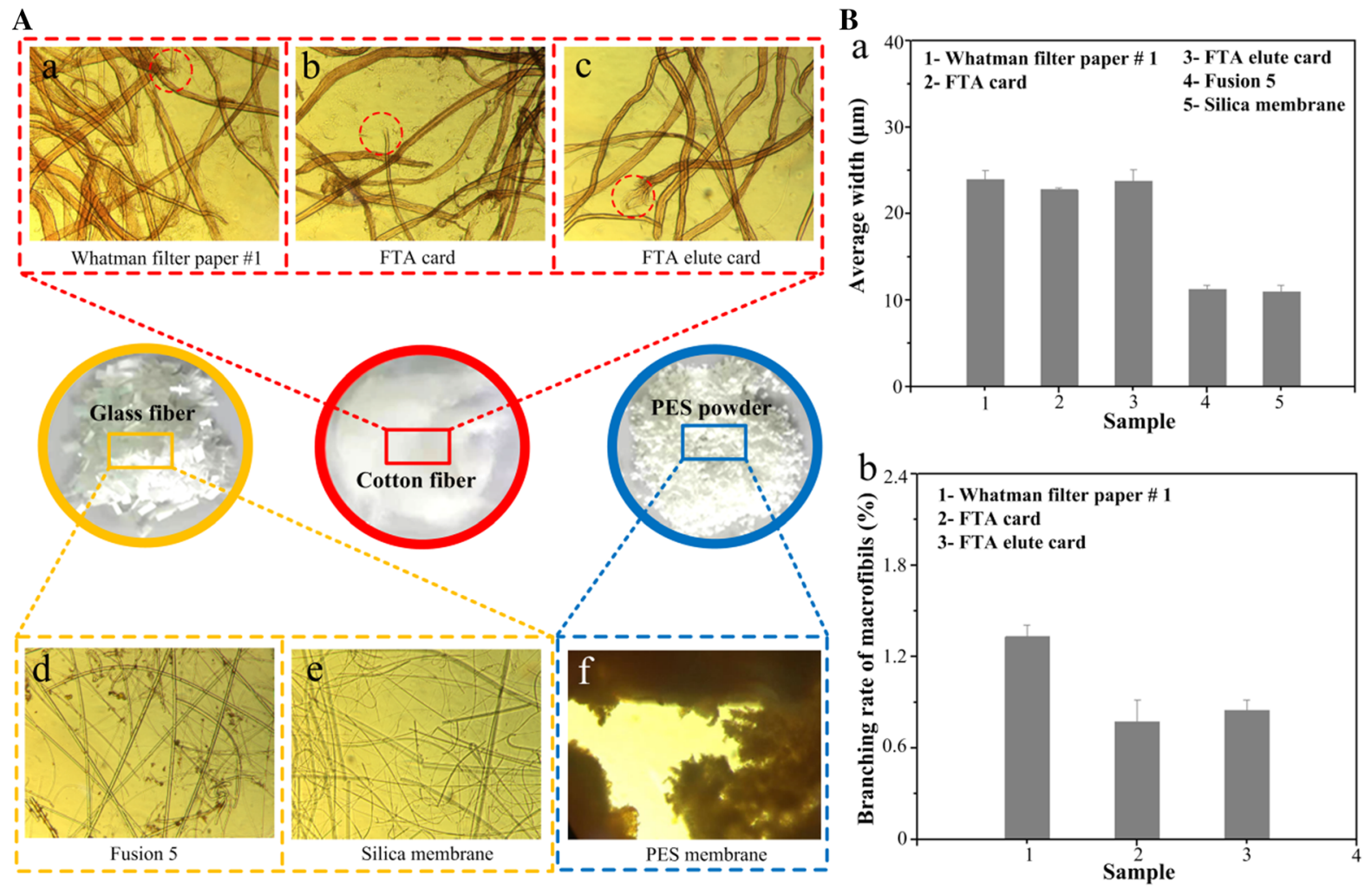

Fig. 2 Fiber type of six paper-based nucleic acid extraction materials. A The morphology of cotton fiber, glass fiber and polymer by optical microscopy; B (a) the average width of cotton fiber and glass fiber and (b) the branching rate of microfibrils of cotton fiber

measured (Table 2). According to the equation (1), the quantity of these materials is FTA elute card $>$ FTA card $>$ silica membrane $>$ Fusion $5>$ Whatman filter paper \#1 > PES membrane, indicating that the FTA elute card needs the most raw materials while PES membrane needs the least; the thickness of these materials is FTA elute card $>$ FTA card $>$ silica membrane $>$ Fusion $5>$ PES membrane $>$ Whatman filter paper \#1. According to the Eq. (2), the tightness of these materials is FTA elute card $>$ Whatman filter paper $\# 1>$ FTA card $>$ PES membrane $>$ Fusion $5>$ silica membrane, indicating that the mechanical properties of cotton fiber-based materials are better than that of polymer-based material, which are better than that of glass fiber-based materials. Additionally, the hydrophilicities of these materials are excellent because the contact angle of each material is zero.

Based on the above analysis, the morphology of these materials was also investigated by SEM (Fig. 3). The front, back and cross section of Whatman filter paper \#1 (Fig. 3A), FTA card (Fig. 3B) and FTA elute card (Fig. 3C) showed that the curved flat fiber arrays are in longitudinal direction and the widths of the fiber are large (the details are as follows: $18.15 \pm 3 \mu \mathrm{m}$ of Whatman filter paper \#1, $17.22 \pm 5.57 \mu \mathrm{m}$ of FTA card and $17.87 \pm 3.62 \mu \mathrm{m}$ of FTA elute card). The

Table 2 The basic physical properties of six paper-based nucleic acid extraction materials

\begin{tabular}{lcrrrrr}
\hline & Whatmanfilter paper\#1 & \multicolumn{1}{l}{ FTA card } & FTA elute card & Fusion 5 & Silica membrane & PESmembrane \\
\hline Quantity $\left(\mathrm{g} / \mathrm{m}^{2}\right)$ & $70.73 \pm 3.54$ & $176.83 \pm 2.82$ & $282.94 \pm 7.07$ & $81.3 \pm 3.49$ & $89.59 \pm 2.39$ & $44.21 \pm 0.7$ \\
Thickness $(\mathrm{mm})$ & $0.18 \pm 0.03$ & $0.5 \pm 0.05$ & $0.64 \pm 0.03$ & $0.4 \pm 0.02$ & $0.49 \pm 0.03$ & $0.19 \pm 0.04$ \\
Tightness $\left(\mathrm{g} / \mathrm{cm}^{3}\right)$ & $0.39 \pm 0.01$ & $0.35 \pm 0.05$ & $0.44 \pm 0.02$ & $0.2 \pm 0.02$ & $0.18 \pm 0.08$ & $0.23 \pm 0.01$ \\
\hline
\end{tabular}



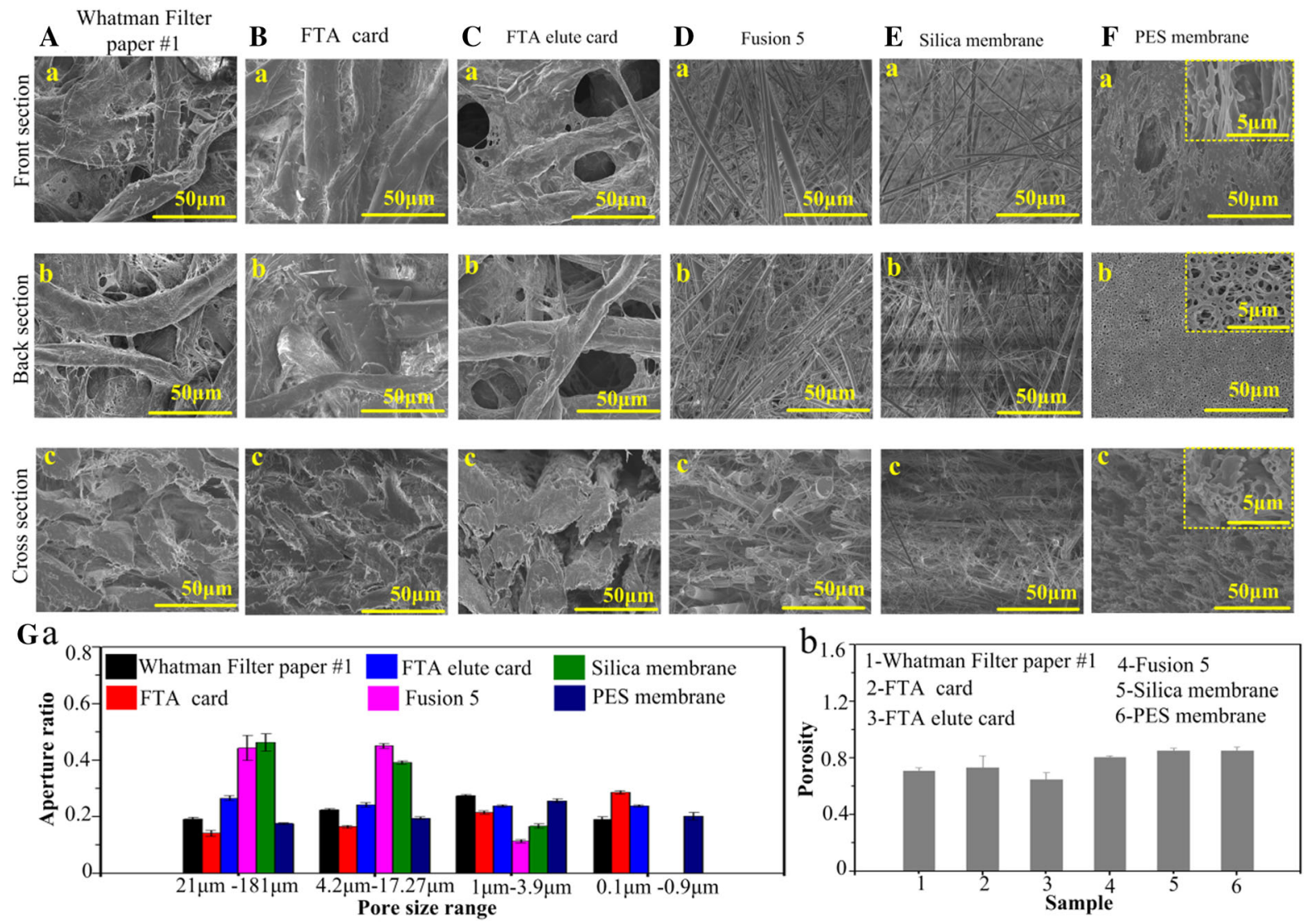

Fig. 3 The morphology of six paper-based nucleic acid extraction materials. The front section, back section and cross section of Whatman filter paper \#1 (A), FTA card (B), FTA elute

fiber is flat from the cross section, indicating that the raw material is cotton fiber as it is consistent with the shape structure of cotton fiber. In addition, the fiber surface of Whatman filter paper \#1 is smooth and has the phenomenon of wire splitting broom (Fig. 3Aa, b), which contributes to the interlacing and winding of fiber to form paper, enhancing the mechanical strength of paper. The fiber surface of FTA card (Fig. 3Ba, b) and FTA elute card (Fig. 3Ca, b) has flaky substances. This maybe the lysis reagent loads on the surface of paper material through chemical modification. These fibers are arranged neatly on the cross section (Fig. 3Ac, Bc, Cc). The front, back and cross sections of Fusion 5 (Fig. 3D) and silica membrane (Fig. 3E) have cylindrical fiber with smooth surface and circular cross section and with small fiber width $(3.75 \pm 2.20$ $\mu \mathrm{m}$ of Fusion 5 and $3.37 \pm 2.09 \mu \mathrm{m}$ of silica membrane), which is similar with the shape structure of glass fiber. The result of PES membrane (Fig. 3F) card (C) Fusion 5 (D), Silica membrane (E) and PES membrane (F). G: a the ratio of different pore sizes and (b) the porosity of six paper-based materials

illustrated that the surface is dense porous structure and there are many cavities in the pore structures from the cross section, indicating that the material has large pore size which is helpful to chemical immobilization of molecules on paper material. However, the structures of front and back section are significantly differences because the volatilization degree of solvent is different on front and back section during the preparation process. This analysis illustrated that PES membrane is made of polymer. Additionally, the pore sizes and porosities of these materials were investigated (Fig. 3G). We observed that the pore size of each material is uneven (Fig. 3Ga). The pore sizes of Whatman filter paper \#1, FTA card and FTA elute card range from 1 to $3.9 \mu \mathrm{m}$, from 0.1 to $0.9 \mu \mathrm{m}$ and from 21 to $181 \mu \mathrm{m}$, respectively, indicating that the adsorption capacity of FTA card is highest and that of FTA elute card is lowest. The pore sizes of Fusion 5, silica membrane and PES membrane range from 21 to 
$181 \mu \mathrm{m}$, from 4.2 to $17.27 \mu \mathrm{m}$ and from 1 to $3.9 \mu \mathrm{m}$, respectively, showing that the adsorption capacity of silica membrane is better than that of Fusion 5, and the adsorption capacity of PES membrane is greater than that of silica membrane. The porosities of these materials are silica membrane $>$ PES membrane $>$ Fusion $5>$ FTA card $>$ Whatman filter paper \#1 $>$ FTA elute card, indicating that the water absorption capability of these materials decreases with the decrease of porosities (Fig. 3Gb).

In NAE process, paper-based material often requires multiple steps operated under water environment, such as immersion in sample, washing with buffer. Thus, the mechanical properties of six paperbased NAE materials were measured by dry tensile strength (Fig. 4A) and wet strength (Fig. 4B). The results indicated that the dry tensile strength of cotton fiber-based material is highest, and that of polymerbased material is lowest, because the surface of cotton fiber has most -OH groups which can increase the combination of cotton fiber. Compared with the dry tensile strengths of these materials, the wet strengths of these materials are significant lower. The result indicated PES membrane $>$ silica membrane $>$ Fusion $5>$ Whatman filter paper \# $1>$ FTA card $>$ FTA elute card, showing that the wet strength of polymer-based material is highest, because polymers are bonded by polymer bonds which are not affected by water; the wet strength of glass fiber-based materials is higher than that of cotton-based materials, because cotton fiber is easy to absorb water and swell in water. The distance between $-\mathrm{OH}$ groups increases after swelled, and the original hydrogen bond breaks and $-\mathrm{OH}$ groups does not combine, resulting in the reduction of the binding force of cotton fiber. Meanwhile, the friction between cotton fibers in water is greatly reduced, thus decreasing the wet strength. In addition, the adhesive force around the glass fiber is weakened after wetted in water, resulting in decrease of the wet strength of glass fiber-based materials. The wet strength of cotton fiber-based materials without modification is higher than that of cotton fiber-based materials with modification, because the friction and adhesion of cotton fiber modified with chemical molecules in water will be further weakened due to the action of chemical molecules on the surface of cotton fiber (Zhang et al. 2014). In addition, the Young's moduli of these materials in dry condition (Fig. 4C) and wet condition (Fig. 4D) is consistent with their dry tensile strength and wet strength. To further observe the wet strength of these materials in real extracted process, these materials were also immersed in water at different times (Fig. 4E). The result showed that the edges of Whatman filter paper \#1, FTA card, FTA elute card, silica membrane and Fusion 5 had burrs and the edge of PES membrane was smooth and did not powder falling within $0 \mathrm{~min}$. After immersion in water for $20 \mathrm{~min}$, the edge of Whatman filter paper \#1 remained unchanged, that is caused by the $-\mathrm{OH}$ functional groups on the surface of this material closely combined (Zhou et al. 2020). The edges of FTA card and FTA elute card had less powder falling, because some chemical reagents were modified on the surface of these materials, which could affect the combination ability of cotton fiber. The edges of Fusion 5 and silica membrane had less glass fiber falling, because the surface of glass fiber has most Si element (it has no combined ability), which affected the interaction combined ability of glass fiber. The edge of PES membrane was still unchanged. Based on above analysis, the wet strengths of these paper-based materials were not significantly changed during immersion in water from $0 \mathrm{~min}$ to $20 \mathrm{~min}$, which could not affect the downstream analysis.

Chemical properties of paper-based nucleic acid extraction materials

In NAE, the chemical properties of paper-based material could help the adsorption capacity of nucleic acid. Based on this consideration, this study firstly measured the surface element of six paper-based NAE materials by EDS (Fig. 5). The result of EDS indicated that the surface of Whatman filter paper \#1 contains $C$ and $\mathrm{O}$ elements (Fig. 5Aa), showing that it is mainly composed of cotton fiber, which is consistent with the result of SEM; the surface of FTA card has $\mathrm{N}$ element besides $\mathrm{C}$ and $\mathrm{O}$ elements (Fig. 5Ab), mainly indicating that the introduction of lysis reagent on the surface of cotton fiber. The surface of FTA elute card contains $\mathrm{C}, \mathrm{N}, \mathrm{O}$ and $\mathrm{S}$ elements (Fig. 5Ac), because lysis reagent and the adsorption reagent of impurities on the surface of cotton fiber contain $\mathrm{N}$ and $\mathrm{S}$ elements, respectively. The surfaces of Fusion 5 (Fig. 5Ad) and silica membrane (Fig. 5Ae) include $\mathrm{Si}$ element, indicating that Fusion 5 and silica membrane are fabricated by glass fiber composed of $\mathrm{SiO}_{2}$. For PES membrane, its surface has $\mathrm{C}, \mathrm{O}$ and $\mathrm{S}$ elements, which 

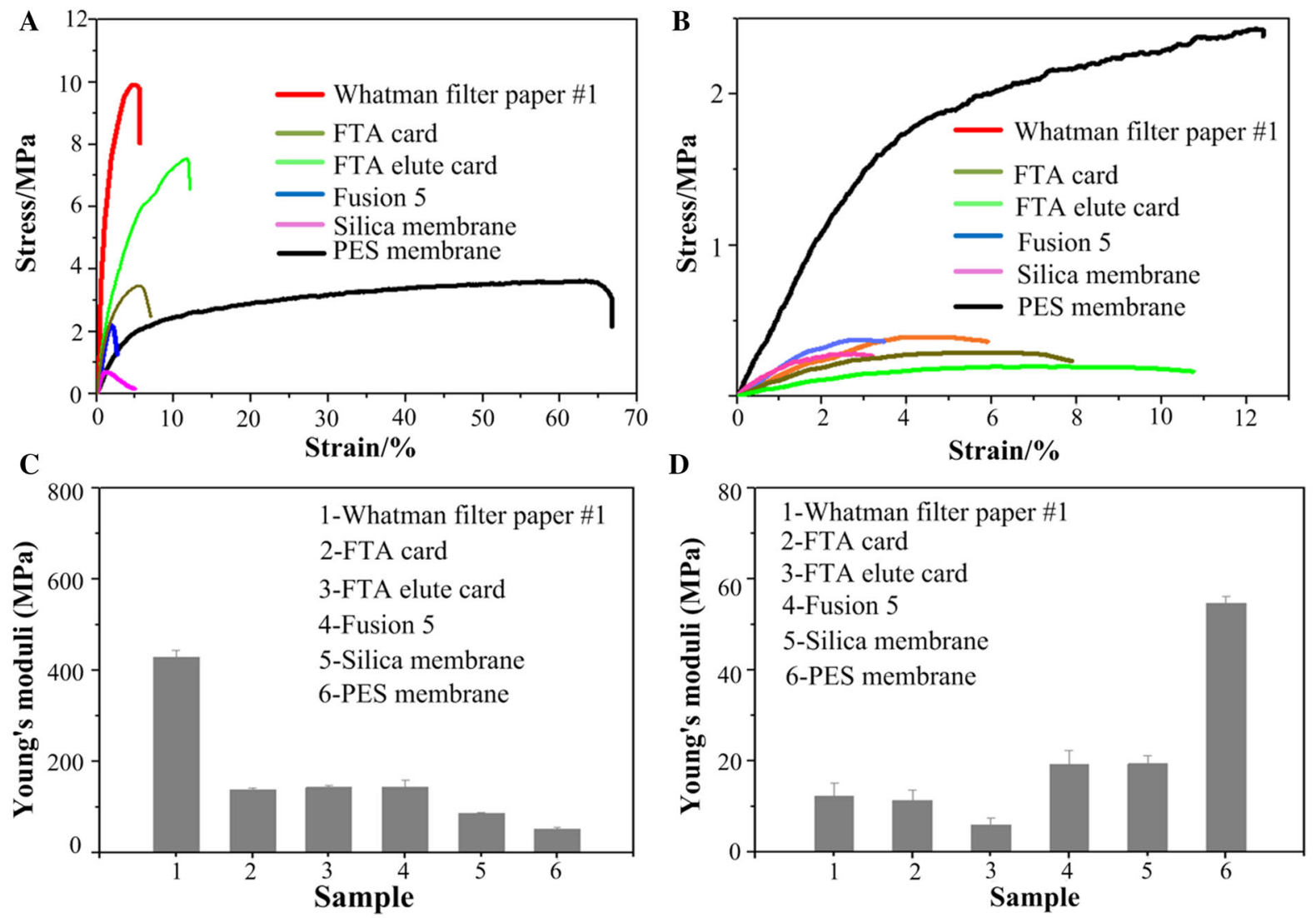

$\mathbf{E}$

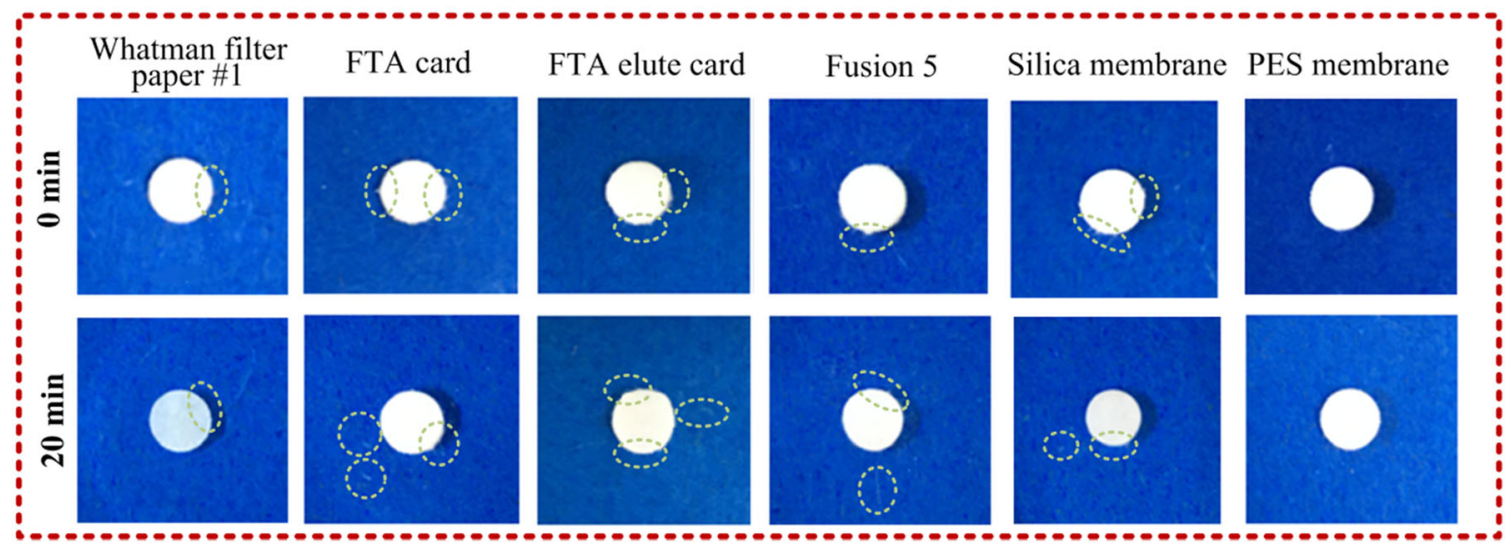

Fig. 4 The mechanical property of six paper-based nucleic acid extraction materials. A The dry strength; B The wet strength; C The Young's modulus of the dry strength; D The Young's

is consistent with the PES polymer (Sile-Yuksel et al. 2014). The weight of element (Fig. 5B) illustrated that the content of element is different because of different compositions of raw materials.

Furthermore, this study also measured the surface functional groups and crystal structure of six paper- modulus of the wet strength; $\mathbf{E}$ The shape changes before and after immersion in water

based NAE materials by FTIR, XPS and XRD (Fig. 6). From the FTIR spectrum of Whatman filter paper \#1, FTA card and FTA elute card (Fig. 6A), the result concluded that the characteristic peak of Whatman filter paper \#1 at 3482, 2906, $1064 \mathrm{~cm}^{-1}$ belongs to the stretching vibration peak of $\mathrm{O}-\mathrm{H}, \mathrm{C}-\mathrm{H}$ and $\mathrm{C}-\mathrm{O}-\mathrm{C}$, 

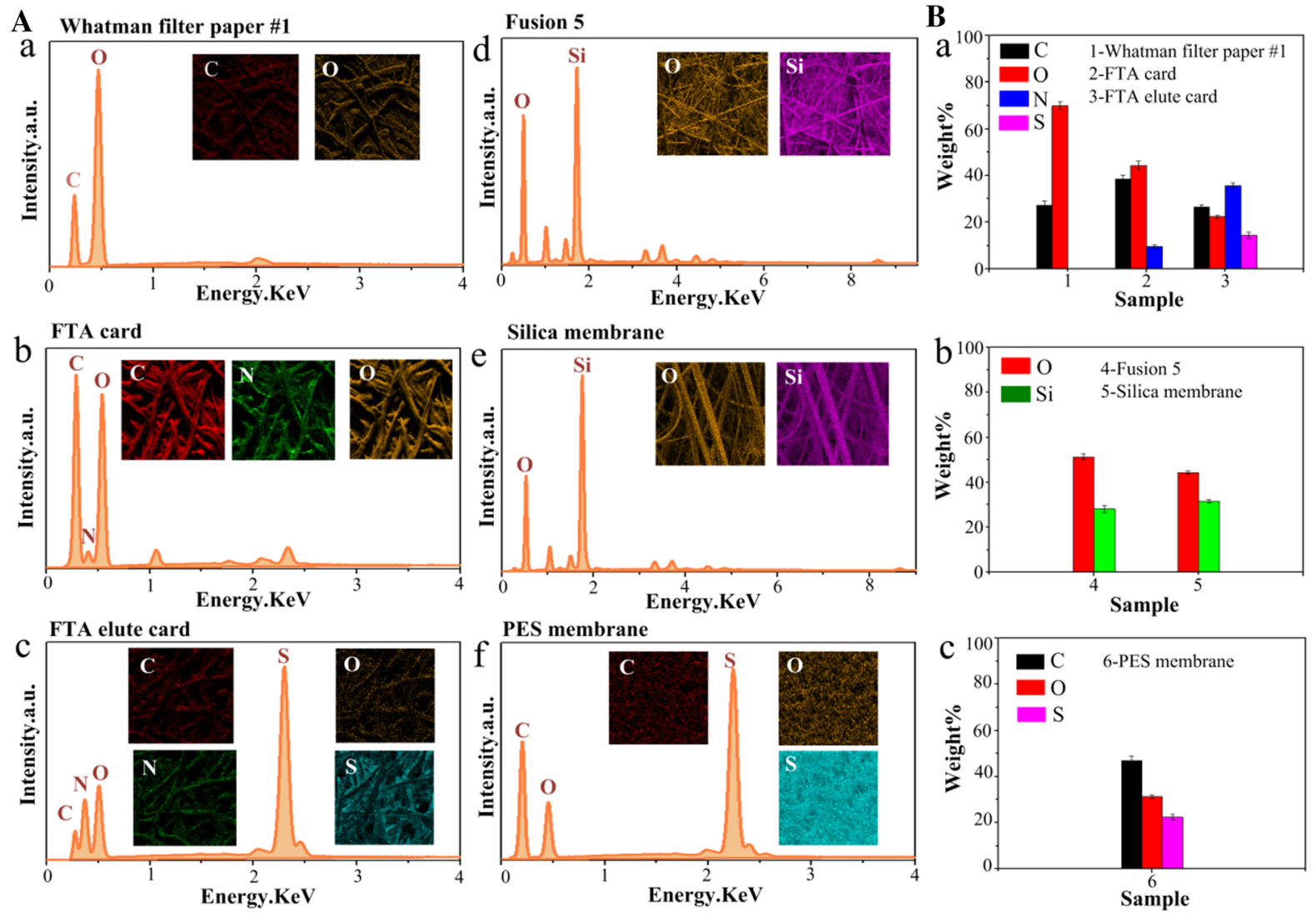

Fig. 5 The surface elements of six paper-based nucleic acid extraction materials. A The element image of six paper-based nucleic acid extraction materials. a Whatman filter paper \#1; b FTA card; c FTA elute card; d Fusion 5; e Silica membrane;

respectively, which is consistent with the structure of cotton fiber. The characteristic peak of FTA card at $3400 \mathrm{~cm}^{-1}$ belongs to the stretching vibration peak of $\mathrm{O}-\mathrm{H}$ and the overlapping vibration peak of $\mathrm{N}-\mathrm{H}$, and the peak at $2909 \mathrm{~cm}^{-1}$ and $1638 \mathrm{~cm}^{-1}$ belongs to the stretching vibration peak of $\mathrm{C}-\mathrm{H}$ and $\mathrm{C}=\mathrm{O}$, respectively. The characteristic peak of FTA elute card at $3433 \mathrm{~cm}^{-1}$ belongs to the stretching vibration peak of $\mathrm{O}-\mathrm{H}$ and the overlapping vibration peak of $\mathrm{N}-\mathrm{H}$, while the peak at $2909 \mathrm{~cm}^{-1}, 2067 \mathrm{~cm}^{-1}, 1638 \mathrm{~cm}^{-1}$, $575 \mathrm{~cm}^{-1}$ belongs to the stretching vibration peak of $\mathrm{C}-$ $\mathrm{H}, \mathrm{C} \equiv \mathrm{N}, \mathrm{C}=\mathrm{O}$ and $\mathrm{C}-\mathrm{S}$. From the FTIR spectrum of Fusion 5, silica membrane and PES membrane (Fig. 6B), the characteristic peak of Fusion 5 at 3620 $\mathrm{cm}^{-1}$ and $1641 \mathrm{~cm}^{-1}$ belongs to the stretching vibration peak of $-\mathrm{OH}$ and the bending vibration peak of $\mathrm{Si}-\mathrm{OH}$; the peak at $1044 \mathrm{~cm}^{-1}$ is the stretching vibration peak of $\mathrm{Si}-\mathrm{O}-\mathrm{Si}$; the peak at $787 \mathrm{~cm}^{-1}$ and $467 \mathrm{~cm}^{-1}$ is the f PES membrane. B The weight of each element. a Whatman filter paper \# 1, FTA card and FTA elute card; b Fusion 5 and Silica membrane; c PES membrane

symmetrical stretching vibration peak of Si-O. The characteristic peak of silica membrane at $3457 \mathrm{~cm}^{-1}$ and $1067 \mathrm{~cm}^{-1}$ belongs to the stretching vibration peak of $-\mathrm{OH}$ and $\mathrm{Si}-\mathrm{O}-\mathrm{Si}$, respectively. The peak at $790 \mathrm{~cm}^{-}$ ${ }^{1}$ and $477 \mathrm{~cm}^{-1}$ belongs to the stretching vibration peak of Si-O. The characteristic peak of PES membrane at $3080 \mathrm{~cm}^{-1}$ is the stretching vibration peak of $\mathrm{C}-\mathrm{H}$ on the benzene ring in PES; the peak at 1321 and 1239 $\mathrm{cm}^{-1}$ is the stretching vibration of C-O-C; the peak at 1150 and $1102 \mathrm{~cm}^{-1}$ is the stretching vibration of $\mathrm{S}=\mathrm{O}$ of sulfone. The above analysis is similar with the results of EDS.

From the result of XPS (Fig. 6C), Whatman filter paper \#1 contains the characteristic peak of $\mathrm{C} 1 \mathrm{~s}$ and $\mathrm{O} 1 \mathrm{~s}$; FTA card has the characteristic peak of C1s, N1s and $\mathrm{O} 1 \mathrm{~s}$, indicating that nitrogenous substances are loaded on the surface of cotton fiber; FTA elute card contains the characteristic peaks of $\mathrm{C} 1 \mathrm{~s}, \mathrm{~N} 1 \mathrm{~s}, \mathrm{O} 1 \mathrm{~s}$ and 

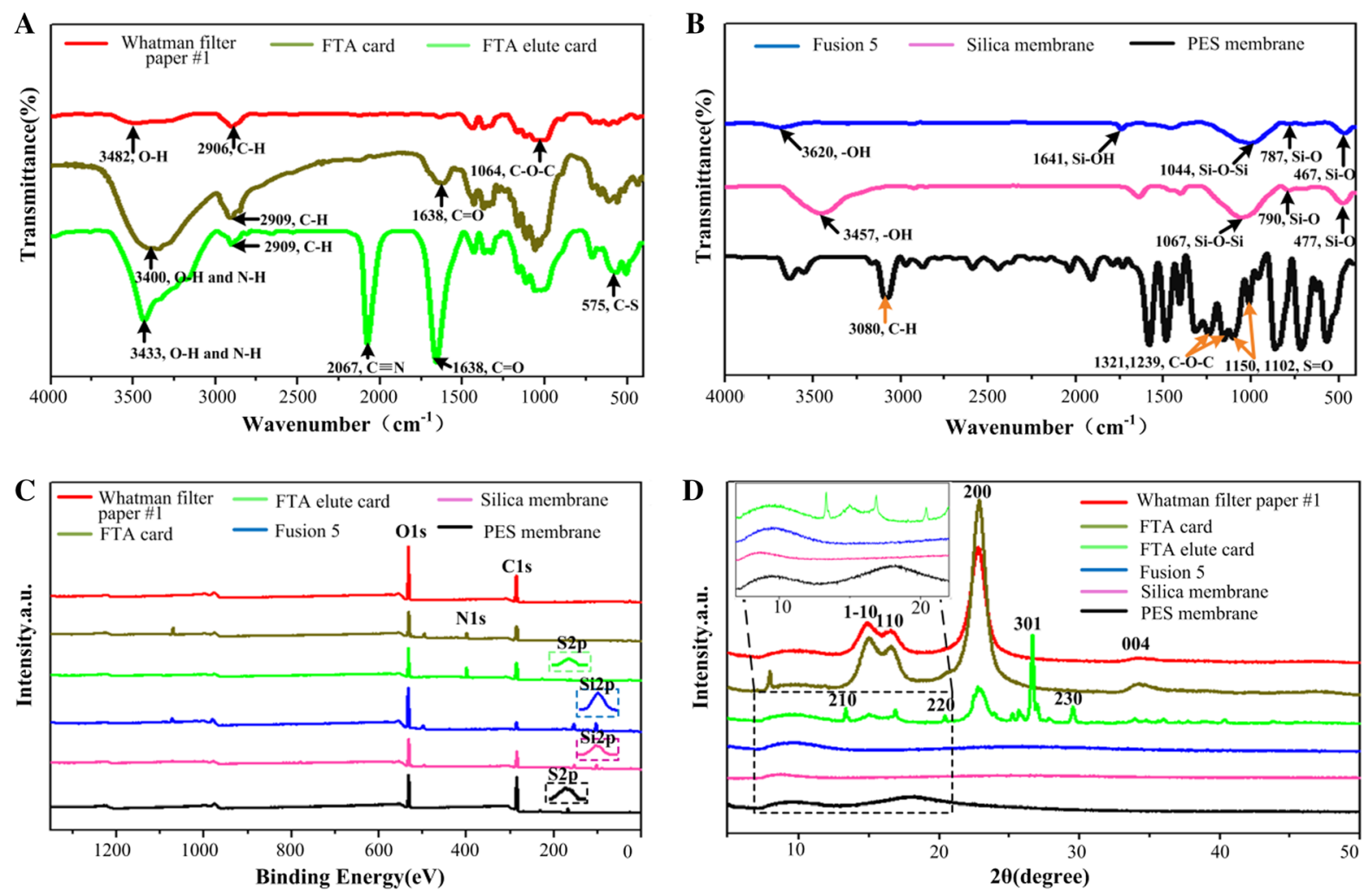

Fig. 6 The functional groups of six paper-based nucleic acid extraction materials. A and B The result of FTIR; C The result of XPS; D The result of XRD.

S2p, showing that nitrogenous and sulfur-containing substances are loaded on the surface of cotton fiber; PES membrane has the characteristic peak of $\mathrm{C} 1 \mathrm{~s}, \mathrm{O} 1 \mathrm{~s}$ and S2p. These results are similar with that of EDS and FTIR. However, Fusion 5 and silica membrane contain the characteristic peaks of O1s, Si2p and C1s, which are not consistent with the corresponding results of EDS and FTIR due to the electron binding energy of exogenous carbon (Swift 1982).

From the crystal structure of XRD (Fig. 6D), the diffraction peak of Whatman filter paper \#1 at $2 \theta=14.8^{\circ}$ corresponds to $1-10$ and $2 \theta=16.5^{\circ}$ corresponds to $110,2 \theta=22.5^{\circ}$ corresponds to 200 , $2 \theta=34.2^{\circ}$ corresponds to 004 , which is consistent with the pattern of filter paper (French 2014), because whatman filter paper wasn't modified with any chemical reagent. The diffraction peak of FTA card is similar with that of Whatman filter paper \# 1, which showed that lysis-loaded reagent doesn't change the crystalline structure of cotton fiber. However, the diffraction peak of FTA elute card at $2 \theta=14.8^{\circ}$, $16.5^{\circ}$ weak, the peak of chaotropic salt at $2 \theta=13.3^{\circ}$, $20.39^{\circ}, 26.6^{\circ}, 29.3^{\circ}$ appears stronger pattern, which indicated that the crystalline region of cellulose is greatly weakened, because the lysis reagent and the adsorption reagent of impurities contains most of chaotropic salt were modified on the surface of FTA elute card, this chaotropic salt maybe change the crystalline structure of cotton fiber. Fusion 5 and silica membrane have amorphous characteristic peak of silica and non-significant diffraction spectrum, which illustrated that there are silica-based materials. Similarly, PES membrane also shows wide peak and nonsignificant diffraction peak, indicating that it is composed of amorphous state polymer.

Validation of the nucleic acid extraction performance

Based on above physical and chemical properties of six paper-based NAE materials, their NAE performances were also evaluated by RT-PCR (quantitative 
detection) and PCR (qualitive detection). In real clinical application, whole blood is a frequently-used clinical sample. Thus, whole blood, as a model, was used to verify the performance of NAE in this study. The amplification curve (Fig. 7A) and standard curve (Fig. 7B) of pure DNA (from $6 \times 10^{0} \mathrm{ng} / \mu \mathrm{L}$ to $6 \times 10^{-3}$ $\mathrm{ng} / \mu \mathrm{L})$ were firstly estabilished. After extracted the same volume $(30 \mu \mathrm{L})$ of whole blood, the amplification curve of RT-PCR is shown in (Fig. 7C). According to the standard curve, the quantity of DNA after extraction is FTA card (3.65 ng) > Qiagen DNA kit $(3.21 \mathrm{ng})>$ Fusion $5(3.05 \mathrm{ng})>$ silica membrane $(2.8$ $\mathrm{ng})>$ FTA elute card $(2.39 \mathrm{ng})>$ Whatman filter paper \# 1 (1.44 ng) > PES membrane (0 ng). The results illustrated that the exraction performance of FTA card is highest as compared to other paper-based materials, while PES membrane is not able to extract DNA from whole blood. Because FTA card needs more washing steps to clean the cell debris and other impurities, indicating that the high-purity DNA was obtained by
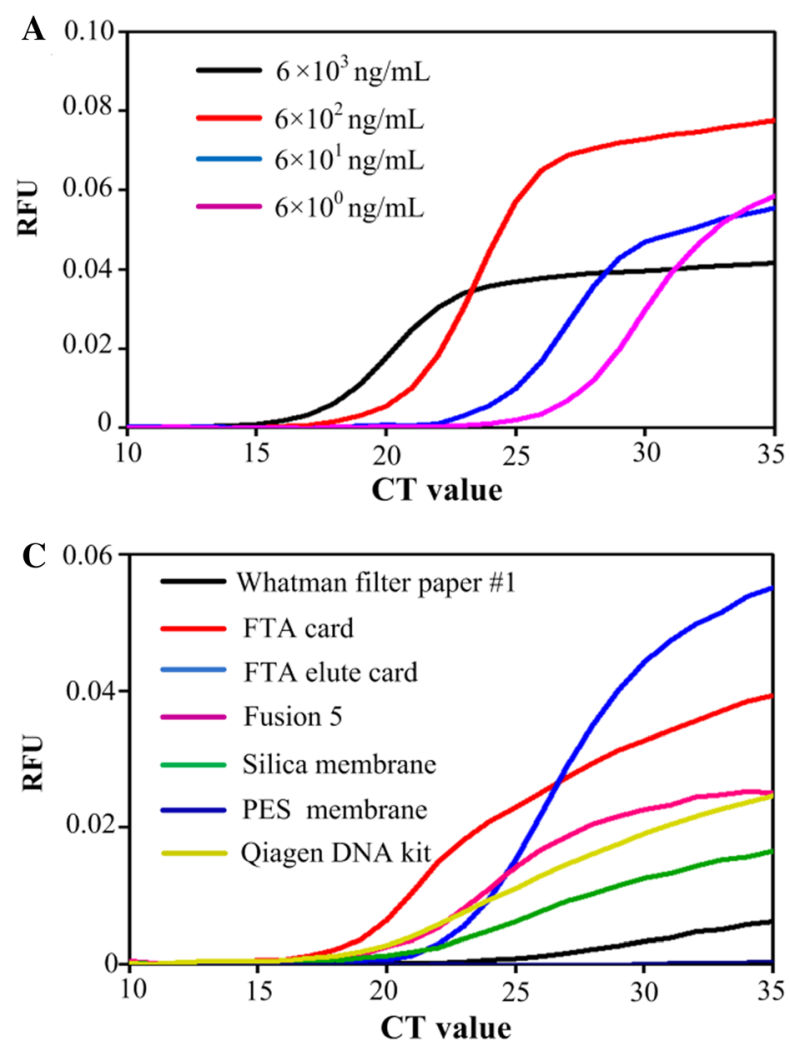

Fig. 7 The quantitative results of six paper-based nucleic acid extraction materials after extraction. A The amplification curve of standard sample; B The standard curve of standard sample (the sample concentration vs the corresponding $\mathrm{Ct}$ ); $\mathbf{C}$ The
FTA card; Qiagen DNA kit uses less elution buffer to elute DNA from spin column, resulting in the loss of DNA; FTA elute card uses less washing steps, resulting in the impurity remains in DNA solution, which maybe affect the purity of DNA; both Fusion 5 and silica membrane use one step method to extract DNA from whole blood without washing steps, which remains most impurities in the DNA solution; Whatman filter paper \#1 does not modify with chemcial reagent and realizes DNA adsorption based on electrostatic adsorption, resulting in the lower adsorption of DNA; the adsorption capacity of PES membrane is lowest, leading to the amplification of RT-PCR/PCR failure. The result of PCR is consistent with that of RT-PCR (Fig. S1). In addition, we also observed the adsorption of impurities of these paper-based materials during their extracted process. As shown in Fig. 7D, the result illustrated that the surfaces of Whatman filter paper \#1, FTA elute card, Fusion 5, PES membrane and silica membrane show pink color,

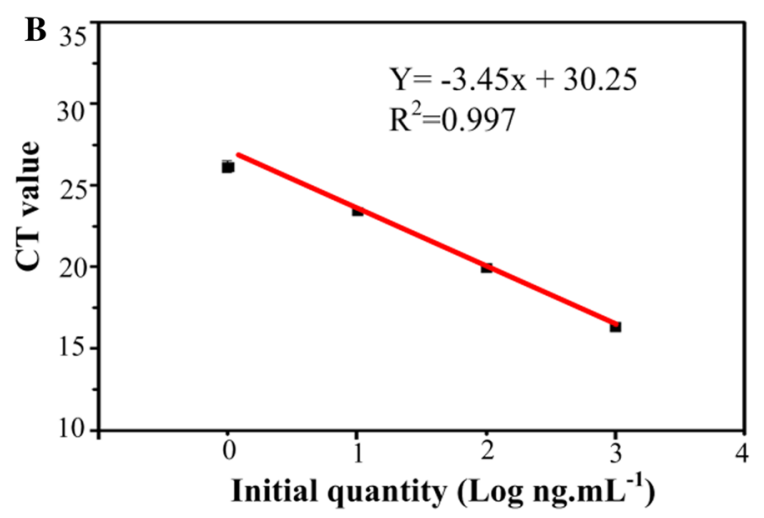

$\mathbf{D}$

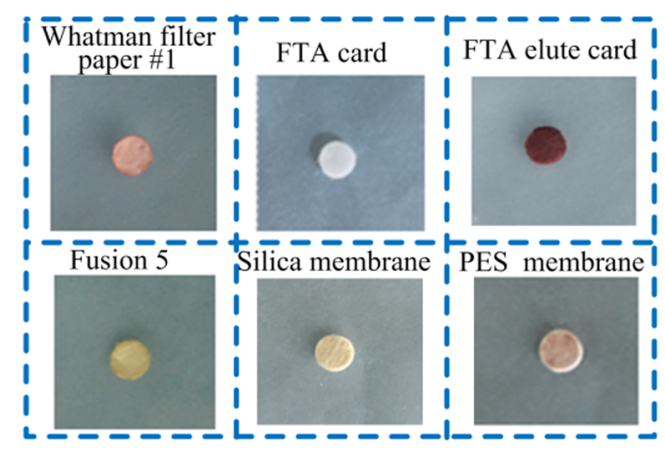

Non-specific adsorption phenomenon

amplification curve of six paper-based nucleic acid extraction materials after extraction. $\mathbf{D}$ The residue condition of cell debris after extraction 
because the non-specific substances (e.g., debris, red cell) remained; the surface of FTA card shows white color, because non-specific substances have been washed.

Through comparison of the extracted process of six paper-based NAE materials, we concluded that Whatman filter paper \#1 needs about 2 min and two steps (lysis step and washing step); FTA card needs $25 \mathrm{~min}$ and three steps (lysis step, first washing step and second washing step); FTA elute card needs $33 \mathrm{~min}$ and five steps (lysis step, washing step, heat step, tapped step and elution step); Fusion 5 and silica membrane require $2 \mathrm{~min}$ and two steps (lysis step and washing step); PES membrane needs $167 \mathrm{~min}$ and five steps (lyisis step, incubation step, adsorption step, washing step and elution step). Qiagen DNA kit needs $18 \mathrm{~min}$ and six steps (mixed step, lysis step, three washing steps, elution step). Based on above analysis, we summarized the differences in Fig. $8 \&$ Table S1 in order to help customer to choose reasonably. From the perspective of practical use, the NAE performance of FTA card is best according to the high concentration and purity of extracted DNA. The comprehensive performance (i.e., cost, time, operation step, extraction efficiency, sample volume) of silica membrane is highest as compared to that of other paper-based materials.
Reasonable suggestions

An ideal paper-based NAE material for POCT application should has characteristics of simple operation, degradability and high efficiency (the high adsorption of nucleic acid and the low adsorption of non-specific substances). Through comparison of the perofrmances of six paper-based NAE materials, PES membrane is not suitable for NAE at POC appliction because of its lowest nucleic acid efficiency. Glass fiber-based material is not eco-friendly due to its non-degradable nature, while cotton fiber-based materials have better performances. Hence, cotton fiber has great potential for preparing novel functional paper-based NAE materials due to its abundance and easy to modification.

Based on the above analysis, there are some shortcomings: (a) the pore sizes of six paper-based NAE materials are heterogeneous, which affect the adsorption efficiency of nucleic acid; (b) the wet strength of cotton fiber-based material with modification (e.g., FTA card, FTA elute card) is lower, which may result in the falling of powder from material edge affecting the adsorption efficiency of nucleic acid; (c) the porosity of cotton fiber-based material is small, indicating that its water adsorption capability is lower thus affecting the purification of nucleic acid during the washing process; (d) the surface of paper-based material has not fucntional groups to reject the non-
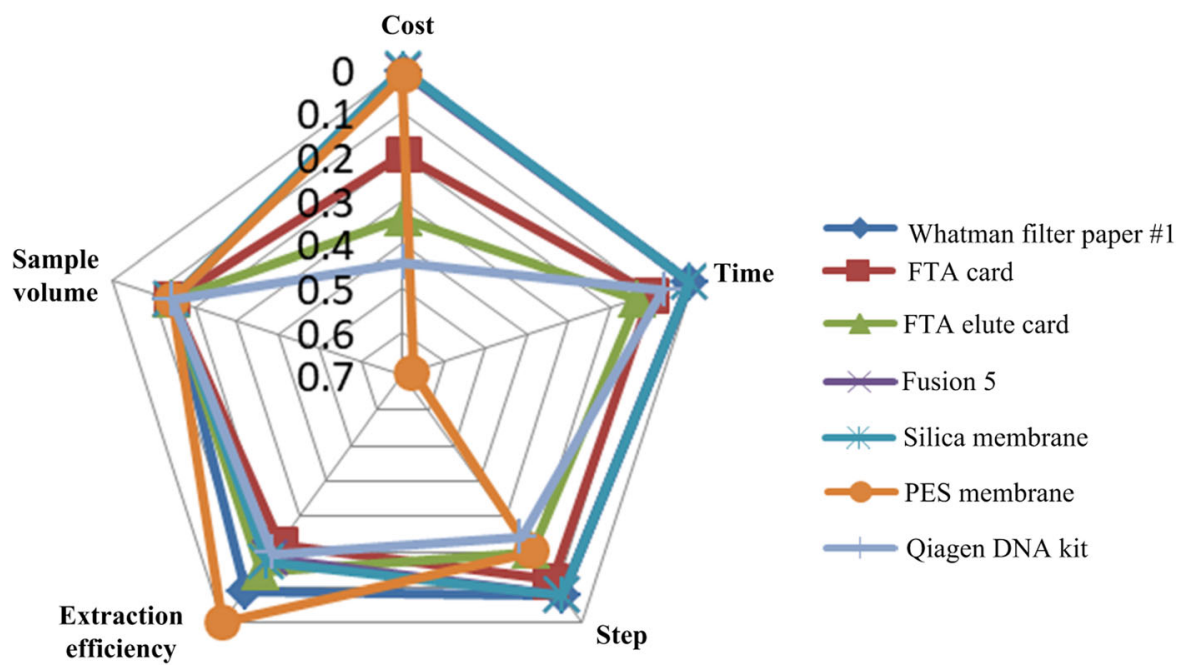

Fig. 8 Comparison of the comprehensive performances of six paper-based nucleic acid extraction materials and Qiagen DNA kit. Note Sample volume: $30 \mu \mathrm{L}$ of whole blood is used for all

materials;Cost:the price per $\phi 4.5 \mathrm{~mm}$; Time: the whole extraction process requires time; Step: operation steps Extraction efficiency: the concentration of extracted nucleic acids 
specific adsorption of impurities besides FTA elute card (FTA elute card needs eultion step), leading to the non-specific protein adsorption.

To make paper-based NAE material achieve the ideal goal, several suggestions are provided: (a) the uniform pore size and the large porosity will be considered in the design and preparation of paperbased material; (b) polymer (e.g., PVA) could be used to mix with cotton fiber to improve the wet strengh of paper-based material; (c) the special functional groups will be grafted on the surface of paper-based material to increase the adsorption of nucleic acid and decrease the non-specific adsorption of impurities at the same time.

To further verify the feasibility of these paperbased materials to other sample, in the future, paperbased materials will be used to extract nucleic acid from some non-invasive body fluids samples such as urine, saliva, vaginal discharge and sewage (Hasandka et al. 2022). In addition, these paper-based materials hold great potential for sample storage, sample concentration and detection. For example, cervial cell sample was stored on FTA card (Pedrao et al. 2021); filter paper was fabricated into origami structure of multiple layers for 3D sequenctial ion concentration polatization of human serum (Lee et al. 2021); filter paper was used to extract cytomegalovirus from urine (Amin et al. 2021).

In addition to paper-based materials, other cellulose-based materials have also been used for nucleic acid extraction and amplification. For example, nylon thread has been used to extract protein-free DNA from whole blood for disease detection (E.coli bacteriophage or lung cancer) (Chen et al. 2021). Additionally, other materials have been immobilized on paper-based materials to achieve various detections. For example, filter paper-based device impregnated with chromogenic substrate reacted with bacterial enzymes to form coloured products for colorimetric detection of S.aureus and E.coli (Asif et al. 2020; Prabhu et al. 2020). Upconversion nanoparticles as a signal reporter was immobilized on test line of nitrocellulose membrane for aflatoxin B1 detection (Guo et al. 2021). Various nanomaterials, such as funtionalized metal nanoparticles and nanozyme, have been used for colorimetric pathogen detection (Nguyen and Kim 2020).

\section{Conclusions}

In this study, the performances of six paper-based NAE materials were compared from their physical properties, chemical properties and the efficiencies of nucleic acid extraction. The characterization of physical properties indicated that uneven pore size, and low wet strength and porosity are not conducive to the adsorption of nucleic acid. The characterization of chemical properties indicated that $\mathrm{N}$ element in paperbased material helps sample lysis, and $\mathrm{Si}$ element contributes to adsorbing nucleic acids while S element is apt to adsorb non-specific substances. The extraction efficiency of FTA card has been demonstrated to be greatest than other paper-based materials. Finally, we envison that paper-based NAE material will play a great role in POCT application.

Acknowledgments This work was supported by the National Natural Science Foundation of China (21808132, 21904104), China Postdoctoral Science Foundation (2018M633525, 2020M673418), Postdoctoral Research Project of Shaanxi Province, China (2018BSHYDZZ75), Fundamental Research Funds for the Central Universities (xzy012019067).

\section{Declarations}

Conflict of interest All authors declare that they have no conflict of interest.

\section{References}

Amin MM, Wong P, McCann M, Dollard SC (2021) Detection of cytomegalovirus in urine dried on filter paper. J Pediat Inf Dis Soc 10(10):958-961. https://doi.org/10.1093/jpids/ piab033

Asif M, Awan FR, Khan QM, Ngamson B, Pamme N (2020) Paper based analytical devices for colorimetric detection of S. aureus and E. coli and their antibiotic resistant strains in milk. Analyst 145(22):7320-7329. https://doi.org/10.1039/ d0an01075h

Batule BS, Seok Y, Kim M-G (2020) Paper-based nucleic acid testing system for simple and early diagnosis of mosquitoborne RNA viruses from human serum. Biosens Bioelectron 151:111998. https://doi.org/10.1016/j.bios.2019. 111998

Chauhan P, Gupta P, Chhikara K, Goyal K, Singh MP (2021) FTA cards for COVID 2019 samples: easy and cost effective innovation. VirusDisease 32(1):20-21. https:// doi.org/10.1007/s13337-021-00677-4

Chen L, Cabot JM, Paull B (2021) Thread-based isotachophoresis for DNA extraction and purification from 
biological samples. Lab Chip 21(13):2565-2573. https:// doi.org/10.1039/d1lc00179e

Chen Y, Liu Y, Shi Y, Ping J, Wu J, Chen H (2020) Magnetic particles for integrated nucleic acid purification, amplification and detection without pipetting. TrAC-Trend Anal Chem 127:115912. https://doi.org/10.1016/j.trac.2020. 115912

Choi JR, Hu J, Tang RH, Gong Y, Feng SS, Ren H, Wen T, Li XJ, Wan Abas WAB, Pingguan-Murphy B, Xu F (2016) An integrated paper-based sample-to-answer biosensor for nucleic acid testing at the point of care. Lab Chip 16(3):611-621. https://doi.org/10.1039/C5LC01388G

Emaus MN, Varona M, Eitzmann DR, Hsieh S-A, Zegar VR, Anderson JL (2020) Nucleic acid extraction: fundamentals of sample preparation methodologies, current advancements, and future endeavors. TrAC-Trend Anal Chem 130:115985. https://doi.org/10.1016/j.trac.2020.115985

Etcheverry M, Barbosa SE (2012) Glass fiber reinforced polypropylene mechanical properties enhancement by adhesion improvement. Materials 5(6):1084-1113. https:// doi.org/10.3390/ma5061084

French AD (2014) Idealized powder diffraction patterns for cellulose polymorphs. Cellulose 21(2):885-896. https:// doi.org/10.1007/s10570-013-0030-4

Gan WP, Zhuang B, Zhang PF, Han JP, Li C-X, Liu P (2014) A filter paper-based microdevice for low-cost rapid, and automated DNA extraction and amplification from diverse sample types. Lab Chip 14(19):3719-3728. https://doi.org/ 10.1039/C4LC00686K

Ghosh R, Hazarika NK (2018) Detection of anti-hepatitis C virus and hepatitis $\mathrm{C}$ virus RNA in dried blood spot specimens using Whatman No. 1 filter paper. Indian J Med Microbi 36(2):230-235. https://doi.org/10.4103/ijmm. IJMM_17_238

Guo XR, Yuan Y, Liu JL, Fu S, Zhang J, Mei QS, Zhang Y (2021) Single-line flow assay platform based on orthogonal emissive upconversion nanoparticles. Anal Chem 93(5):3010-3017. https://doi.org/10.1021/acs.analchem. $0 \mathrm{c} 05061$

Hasandka A, Singh AR, Prabhu A, Singhal HR, Nandagopal MSG, Mani NK (2022) Paper and thread as media for the frugal detection of urinary tract infections (UTIs). Anal Bioanal Chem 414:847-865. https://doi.org/10.1007/ s00216-021-03671-3

Hashimoto M, Bando M, Kido J-I, Yokota K, Mita T, Kajimoto K, Kataoka M (2019) Nucleic acid purification from dried blood spot on FTA Elute Card provides template for polymerase chain reaction for highly sensitive plasmodium detection. Parasitol Int 73:101941. https://doi.org/10.1016/ j.parint.2019.101941

Jangam SR, Yamada DH, McFall SM, Kelso DM (2009) Rapid, point-of-care extraction of human immunodeficiency virus type 1 proviral DNA from whole blood for detection by real-time PCR. J Clin Microbiol 47(8):2363-2368. https:// doi.org/10.1128/JCM.r00092-09

Jiang B, Zhang N, Wang BY, Yang N, Huang ZH, Yang HW, Shu ZJ (2018) Deep eutectic solvent as novel additive for PES membrane with improved performance. Sep Purif Technol 194:239-248. https://doi.org/10.1016/j.seppur. 2017.11.036
Lee J, Yoo YK, Lee D, Kim C, Kim KH, Lee S, Kwak S, Kang JY, Kim H, Yoon DS, Hur D, Lee JH (2021) Origami paper-based sample preconcentration using sequentially driven ion concentration polarization. Lab Chip 21(5):867-874. https://doi.org/10.1039/d01c01032d

Li ZD, Bai YM, You ML, Hu J, Yao CY, Cao L, Xu F (2021) Fully integrated microfluidic devices for qualitative, quantitative and digital nucleic acids testing at point of care. Biosens Bioelectron 177:112952. https://doi.org/10. 1016/j.bios.2020.112952

Li ZJ, Liu FF, Cui JZ, Peng ZB, Chang ZR, Lai SJ, Chen QL, Wang LP, Gap GF, Feng ZJ (2021) Comprehensive largescale nucleic acid-testing strategies support China's sustained containment of COVID-19. Nat Med 27(5):740-742. https://doi.org/10.1038/s41591-02101308-7

Mandal G, Das S, Padmanabhan S (2018) Development of a membrane-based method for isolation of genomic DNA from human blood. J Biomol Tech 29(2):46-53. https://doi. org/10.7171/jbt.18-2902-001

Mason MG, Botella JR (2020) Rapid (30-second), equipmentfree purification of nucleic acids using easy-to-make dipsticks. Nat Protoc 15(11):3663-3677. https://doi.org/10. 1038/s41596-020-0392-7

Mika-Gospodorz B, Giengkam S, Westermann AJ, Wongsantichon J, Kion-Crosby W, Chuenklin S, Wang LC, Sunyakumthorn P, Sobota RM, Subbian S, Vogel J, Barquist L, Salje J (2020) Dual RNA-seq of orientia tsutsugamushi informs on host-pathogen interactions for this neglected intracellular human pathogen. Nat Commun 11(1):1-14. https://doi.org/10.1038/s41467-020-17094-8

Nguyen QH, Kim MI (2020) Nanomaterial-mediated paperbased biosensors for colorimetric pathogen detection. TrAC-Trend Anal Chem 132:116038. https://doi.org/10. 1016/j.trac.2020.116038

Pedrao PG, de Carvalho AC, Possati-Resende JC, de Paula CF, Campanella NC, de Oliverira CM, Tavares Guerreiro Fregnani JH (2021) DNA recovery using ethanol-based liquid medium from FTA card-stored samples for HPV detection. Acta Cytol 65(3):264-271. https://doi.org/10. $1159 / 000515913$

Prabhu A, Nandagopal MSG, Yegneswaran PP, Singhal HR, Mani NK (2020) Inkjet printing of paraffin on paper allows low-cost point-of-care diagnostics for pathogenic fungi. Cellulose 27(13):7691-7701. https://doi.org/10.1007/ s10570-020-03314-3

Rodriguez NM, Linnes JC, Fan A, Ellenson CK, Pollock NR, Klapperich CM (2015) Paper-based RNA extraction, in situ isothermal amplification, and lateral flow detection for low-cost, rapid diagnosis of influenza A (H1N1) from clinical specimens. Anal Chem 87(15):7872-7879. https:// doi.org/10.1021/acs.analchem.5b01594

Sile-Yuksel M, Tas B, Koseoglu-Imer DY, Koyuncu I (2014) Effect of silver nanoparticle (AgNP) location in nanocomposite membrane matrix fabricated with different polymer type on antibacterial mechanism. Desalination 347:120-130. https://doi.org/10.1016/j.desal.2014.05.022

Swift P (1982) Adventitious carbon-the panacea for energy referencing. Surf Interface Anal 4(2):47-51. https://doi. org/10.1002/sia.740040204 
Tang RH, Liu LN, Zhang SF, He XC, Li XJ, Xu F, Ni YH, Li F (2019) A review on advances in methods for modification of paper supports for use in point-of-care testing. Microchim Acta 186(8):1-25. https://doi.org/10.1007/s00604019-3626-z

Tang RH, Yang H, Choi JR, Gong Y, Hu J, Wen T, Li XJ, Xu B, Mei QB, Xu F (2017) Paper-based device with on-chip reagent storage for rapid extraction of DNA from biological samples. Microchim Acta 184(7):2141-2150. https:// doi.org/10.1007/s00604-017-2225-0

Wang XD, Yan CY, Wang XK, Zhao XL, Shi C, Ma CP (2020) Integrated silica membrane-based nucleic acid purification, amplification, and visualization platform for low-cost, rapid detection of foodborne pathogens. Anal Bioanal Chem 412(25):6927-6938. https://doi.org/10.1007/ s00216-020-02823-1

Xun GH, Lane ST, Petrov VA, Pepa BE, Zhao HM (2021) A rapid, accurate, scalable, and portable testing system for COVID-19 diagnosis. Nat Commun 12(1):1-9. https://doi. org/10.1038/s41467-021-23185-x

Yang L, Kim TH, Cho HY, Luo J, Lee JM, Chueng STD, Hou YN, Yin PTT, Han JY, Kim JH, Chung BG, Choi JW, Lee KB (2021) Hybrid graphene-gold nanoparticle-based nucleic acid conjugates for cancer-specific multimodal imaging and combined therapeutics. Adv Funct Mater 31(5):2006918. https://doi.org/10.1002/adfm.202006918

Yi CY, Luo ZS, Lu YT, Belwal T, Pan XL, Lin XY (2021) Nanoporous hydrogel for direct digital nucleic acid amplification in untreated complex matrices for single bacteria counting. Biosens Bioelectron 184:113199. https://doi.org/10.1016/j.bios.2021.113199

Zou YP, Mason MG, Wang YL, Wee E, Turni C, Blackall PJ, Trau M, Botella JR (2017) Nucleic acid purification from plants, animals and microbes in under 30 seconds. Plos biol 15(11):e2003916. https://doi.org/10.1371/journal.pbio. 2003916

Zhang Y, Li HF, Li XD, Gibril ME, Yu MH (2014) Chemical modification of cellulose by in situ reactive extrusion in ionic liquid. Carbohyd Polym 99:126-131. https://doi.org/ 10.1016/j.carbpol.2013.07.084

Zhao DW, Zhu Y, Cheng WK, Chen WS, Wu YQ, Yu HP (2020) Cellulose-based flexible functional materials for emerging intelligent electronics. Adv Mater 33(28):2000619. https:// doi.org/10.1002/adma.202000619

Zhou XL, Fu YQ, Chen LD, Wang RB, Wang X, Miao YC, Ji XX, Bian HY, Dai HQ (2020) Diisocyanate modifiable commercial filter paper with tunable hydrophobicity, enhanced wet tensile strength and antibacterial activity. Carbohyd Polym 248:116791. https://doi.org/10.1016/j. carbpol.2020.116791

Publisher's Note Springer Nature remains neutral with regard to jurisdictional claims in published maps and institutional affiliations. 